\title{
Conformations and three-dimensional structures of selected SARS-CoV-2 drug candidates.
}

\author{
Karl N. Kirschner ${ }^{1,}{ }^{*}$, Javed Razzaq ${ }^{1}$, Rudolf Berrendorf ${ }^{1}$, and Wolfgang Heiden ${ }^{1}$ \\ ${ }^{1}$ Department of Computer Science, University of Applied Sciences Bonn-Rhein-Sieg, Grantham-Allee 20, \\ 53757 Sankt Augustin, Germany
}

April 4, 2020

\begin{abstract}
Quantum mechanical theories are used to search and optimize the conformations of proposed small molecule candidates for treatment of SARS-CoV-2. These candidate compounds are taken from what is reported in the news and in other pre-peer-reviewed literature (e.g. ChemRxiv, bioRxiv). The goal herein is to provide predicted structures and relative conformational stabilities for selected drugs and ligands, in the hopes that other research groups can make use of them for developing a treatment.

Initial exploration for conformations are performed at the $\mathrm{HF} / 6-31 \mathrm{G}(\mathrm{d}) / / \mathrm{HF} / 6-31 \mathrm{G}(\mathrm{d})$ theory level, which are then further optimized at more rigorous theory levels (e.g. B97-D3BJ/ccpVTZ//B97-D3BJ/cc-pVTZ). The resulting structures are made available via GitHub.

Please note that we are not advocating that the compounds focused herein should be used as medical treatments for the SARSCoV-2. Instead we are simply providing predicted conformations and relative energies of compounds that can be used for further research purposes.
\end{abstract}

\section{Introduction}

Across the world researchers are focused upon finding a drug treatment for the Coronavirus Disease-2019 (SARS-CoV-2). Logically, much of the focus is repurposing approved drugs, followed by those that are in drug development pipeline. Repurposing drugs is a much quicker endeavor than discovering new ones since their chemical optimization, toxicology profiling, clinical trials and bulk manufacturing are already in place [1]. Several research groups have already proposed possible candidates for testing [2-7]. The results of this testing could provide a spectrum of outcomes - ranging from a compound that show high promise for use in patient treatment to very little activity. However, hindering some our understanding will be the lack of three-dimensional (3D) knowledge of the drugs' structures and their conformations.

Promiscuity underlies the concept of drug repurposing [8-13]. A promiscuous drug (i.e. ligand) implies that it is structurally and chemically complementary to several receptor, while a promiscuous receptor implies that several ligands possess high similarity.

\footnotetext{
*Corresponding author: karl.kirschner@h-brs.de
}

Limiting our ability to exploit ligand similarity for repurposing are the difficulties to experimentally elucidate or theoretically predict 3D conformations and their electrostatic profiles. This difficulty arises from the dynamic nature of molecules and their complex multidimensional potential energy surface [14]. Affirming this is the fact that only a fraction of the 1634 approved small-molecule drugs [15] have a single 3D conformation resolved.

Herein, the 3D structures of the top candidates for SARS-CoV-2 treatment are computed using quantum mechanical (QM) theories, starting with chloroquine since it is already being tested in hospitals and proceeding to other approved drugs according to Table 1 in reference [4]. An semi-extensive conformational search is performed for each molecule in hopes that the low energy conformations are well represented. Since this work is ongoing, we provide the coordinates of the conformations via GitHub (https://github.com/karlkirschner/SARS-CoV-2-3D-Structures) to allow other researchers immediate access to the data.

\section{Methodology}

Initial structures were obtained from the Protein Data Bank [16] when and experimentally determined geometry is available, or from ChemPub [17]. Full geometry optimizations were performed at the $\mathrm{HF} / 6-31 \mathrm{G}(\mathrm{d}) / / \mathrm{HF} / 6-31 \mathrm{G}(\mathrm{d})$ theory level. Optimizations at more rigorous theory levels will be performed (e.g. B97-D3BJ/ccpVTZ and MP2/cc-pVTZ) as time allows.

Molecules studied:

- chloroquine [18] (model building): 2 configurational stereoisomer

- silmitasertib [19] (initial structure: 3NGA X-ray): single topology

- valproic acid [20]: (initial structure: 1DIT X-ray): single topology

All QM calculations were done using Psi4 (v. 1.1a2.dev170) [21]. Figures were created using PyMol [22], Python3, Matplotlib [23] and Inkscape. 


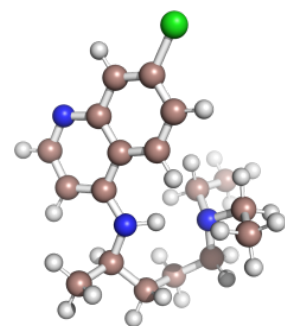

a

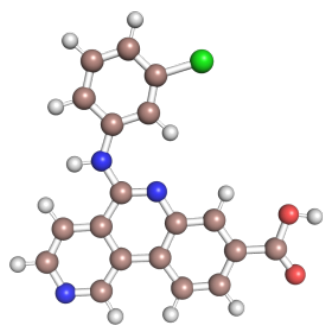

b

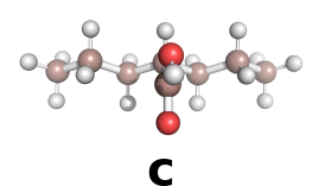

Figure 1: Geometries of the most stable minima identified using B97-D3BJ/cc-pVTZ//B97-D3BJ/cc-pVTZ for a) Chloroquine isomer $1 \mathrm{~b}$ ) Silmitasertib and c) Valproic acid.

\section{Results and Discussion}

The HF/6-31G(d)//HF/6-31G(d) theory level performs fairly well for generating optimized geometries and is relatively inexpensive with regards to computational cost. The use of this theory allows for a quick preview of the possible conformations that a given molecule might have. However, past experience has shown that it often over predicts the number of minima in comparison to an electron-correlated theory level and larger basis set (e.g. B97-D3BJ/cc-pVTZ, MP2/cc-pVTZ). Due to the quick need of structural data, a majority of the initial calculations will be done at HF/6-31G(d)//HF/6-31G(d) theory level. The resulting structures should be an adequate starting point for our initial understanding of how structure might influence the activity observed experimentally. Alternatively, these structures can also be used for computational docking studies and for building molecular dynamics models if desired. However, optimizations are continually being completed at higher theory levels. When these are completed, these geometries should preferentially be considered.

All currently optimized geometries can be obtained following the GitHub link mentioned in the Introduction. Each xyzformatted file contains all of the optimized conformations, ordered from most- to least-stable conformation.

The relative energies for a given molecule (and isomer when relevant) are provided in Tables 1-4. Concerning relative stability of the conformations, $\mathrm{HF} / 6-31 \mathrm{G}(\mathrm{d}) / / \mathrm{HF} / 6-31 \mathrm{G}(\mathrm{d})$ is able to provide a very general ordering of the predicted relative energies. However, including electron correlation into the calculations with at least a triple-zeta basis set is required for more reliable predictions of relative energies. As time allows, more refined optimizations will be performed using more rigorous theory levels (e.g. B97D3BJ/cc-pVTZ, MP2/cc-pVTZ). The tables within the body of the manuscript represent the most rigorous theory levels currently computed.

Upon request, partial atomic charges for these structures can also be determined relatively quickly using the AM1-BCC or RESP methodologies.

\section{Acknowledgment}

The UBRS's computer hardware was supported by the Federal Ministry for Education and Research and by the Ministry for Innovation, Science, Research, and Technology of the state Northrhine-Westfalia (research grant 13FH156IN6).

\section{References}

[1] T. T. Ashburn and K. B. Thor, "Drug repositioning: identifying and developing new uses for existing drugs," Nat Rev Drug Discov, vol. 3, pp. 673-683, Aug. 2004.

[2] Y. W. Chen, C.-P. Yiu, and K.-Y. Wong, "Prediction of the SARS-CoV-2 (2019-nCoV) 3C-like Protease (3CLpro) Structure: Virtual Screening Reveals Velpatasvir, Ledipasvir, and Other Drug Repurposing Candidates," ChemRxiv, DOI:10.26434/chemrxiv.11831103.v2, 22020.

[3] A. Contini, "Virtual Screening of an FDA Approved Drugs Database on Two COVID-19 Coronavirus Proteins," ChemRxiv, DOI:10.26434/chemrxiv.11847381.v1, 2020.

[4] D. E. Gordon, G. M. Jang, M. Bouhaddou, J. Xu, K. Obernier, M. J. O’Meara, J. Z. Guo, D. L. Swaney, T. A. Tummino, R. Huttenhain, R. Kaake, A. L. Richards, B. Tutuncuoglu, H. Foussard, J. Batra, K. Haas, M. Modak, M. Kim, P. Haas, B. J. Polacco, H. Braberg, J. M. Fabius, M. Eckhardt, M. Soucheray, M. Brewer, M. Cakir, M. J. McGregor, Q. Li, Z. Z. C. Naing, Y. Zhou, S. Peng, I. T. Kirby, J. E. Melnyk, J. S. Chorba, K. Lou, S. A. Dai, W. Shen, Y. Shi, Z. Zhang, I. Barrio-Hernandez, D. Memon, C. Hernandez-Armenta, C. J. Mathy, T. Perica, K. B. Pilla, S. J. Ganesan, D. J. Saltzberg, R. Ramachandran, X. Liu, S. B. Rosenthal, L. Calviello, S. Venkataramanan, Y. Lin, S. A. Wankowicz, M. Bohn, R. Trenker, J. M. Young, D. Cavero, J. Hiatt, T. Roth, U. Rathore, A. Subramanian, J. Noack, M. Hubert, F. Roesch, T. Vallet, B. Meyer, K. M. White, L. Miorin, D. Agard, M. Emerman, D. Ruggero, A. Garc\&amp;iacute-Sastre, N. Jura, M. von Zastrow, J. Taunton, O. Schwartz, M. Vignuzzi, C. d'Enfert, S. Mukherjee, M. Jacobson, H. S. Malik, D. G. Fujimori, T. Ideker, C. S. Craik, S. Floor, J. S. Fraser, J. Gross, A. Sali, T. Kortemme, P. Beltrao, K. Shokat, B. K. Shoichet, and N. J. Krogan, "A sars-cov-2-human protein-protein interaction map reveals drug targets and potential drug-repurposing," bioRxiv, DOI: 10.1101/2020.03.22.002386, 2020.

[5] N. Shaghaghi, "Molecular Docking Study of Novel COVID19 Protease with Low Risk Terpenoides Compounds of Plants," ChemRxiv, DOI:10.26434/chemrxiv.11935722.v1, 3 2020.

[6] M. Smith and J. C. Smith, "Repurposing Therapeutics for COVID-19: Supercomputer-Based Docking to the SARSCoV-2 Viral Spike Protein and Viral Spike Protein-Human ACE2 Interface," 32020.

[7] J. Wang, "Fast Identification of Possible Drug Treatment of Coronavirus Disease -19 (COVID-19) Through Com- 
Table 1: Chloroquine isomer 1's relative energies $\left(\mathrm{kcal} \cdot \mathrm{mol}^{-1}\right)$ computed at B97-D3BJ/cc-pVTZ//B97-D3BJ/cc-pVTZ theory level.

\begin{tabular}{lc|lc|lc}
\hline \hline Conf. & $\Delta \mathrm{E}$ & Conf. & $\Delta \mathrm{E}$ & Conf. & $\Delta \mathrm{E}$ \\
\hline 1 & 0.000 & 35 & 8.627 & 69 & 10.719 \\
2 & 1.560 & 36 & 8.627 & 70 & 10.785 \\
3 & 1.958 & 37 & 8.638 & 71 & 10.901 \\
4 & 2.085 & 38 & 8.646 & 72 & 10.990 \\
5 & 3.697 & 39 & 8.797 & 73 & 11.031 \\
6 & 4.339 & 40 & 8.868 & 74 & 11.094 \\
7 & 4.358 & 41 & 9.059 & 75 & 11.126 \\
8 & 4.918 & 42 & 9.115 & 76 & 11.158 \\
9 & 5.566 & 43 & 9.150 & 77 & 11.177 \\
10 & 5.911 & 44 & 9.168 & 78 & 11.240 \\
11 & 6.339 & 45 & 9.168 & 79 & 11.286 \\
12 & 6.863 & 46 & 9.172 & 80 & 11.460 \\
13 & 7.142 & 47 & 9.201 & 81 & 11.527 \\
14 & 7.253 & 48 & 9.245 & 82 & 11.594 \\
15 & 7.303 & 49 & 9.274 & 83 & 11.905 \\
16 & 7.370 & 50 & 9.303 & 84 & 11.927 \\
17 & 7.448 & 51 & 9.309 & 85 & 11.964 \\
18 & 7.534 & 52 & 9.429 & 86 & 11.964 \\
19 & 7.557 & 53 & 9.531 & 87 & 11.991 \\
20 & 7.603 & 54 & 9.583 & 88 & 12.043 \\
21 & 7.639 & 55 & 9.594 & 89 & 12.075 \\
22 & 7.752 & 56 & 9.611 & 90 & 12.079 \\
23 & 7.779 & 57 & 9.655 & 91 & 12.084 \\
24 & 7.866 & 58 & 10.003 & 92 & 12.138 \\
25 & 7.961 & 59 & 10.068 & 93 & 12.381 \\
26 & 8.049 & 60 & 10.153 & 94 & 12.656 \\
27 & 8.147 & 61 & 10.156 & 95 & 13.011 \\
28 & 8.217 & 62 & 10.200 & 96 & 13.162 \\
29 & 8.279 & 63 & 10.226 & 97 & 13.308 \\
30 & 8.347 & 64 & 10.231 & 98 & 13.417 \\
31 & 8.348 & 65 & 10.301 & 99 & 13.590 \\
32 & 8.408 & 66 & 10.403 & 100 & 13.957 \\
33 & 8.421 & 67 & 10.411 & 101 & 14.119 \\
34 & 8.536 & 68 & 10.423 & 102 & 14.490 \\
\hline \hline & & & & & \\
\hline
\end{tabular}


Table 2: Chloroquine isomer 2's relative energies $\left(\mathrm{kcal} \cdot \mathrm{mol}^{-1}\right)$ computed at $\mathrm{HF} / 6-31 \mathrm{G}(\mathrm{d}) / / \mathrm{HF} / 6-31 \mathrm{G}(\mathrm{d})$ theory level.

\begin{tabular}{lc|lc|lc|lc|cc|cc}
\hline \hline Conf. & $\Delta \mathrm{E}$ & Conf. & $\Delta \mathrm{E}$ & Conf. & $\Delta \mathrm{E}$ & Conf. & $\Delta \mathrm{E}$ & Conf. & $\Delta \mathrm{E}$ & Conf. & $\Delta \mathrm{E}$ \\
\hline 1 & 0.000 & 20 & 2.398 & 39 & 3.966 & 58 & 4.882 & 76 & 5.973 & 94 & 8.504 \\
2 & 0.529 & 21 & 2.570 & 40 & 3.975 & 59 & 4.917 & 77 & 6.222 & 95 & 8.610 \\
3 & 0.653 & 22 & 2.647 & 41 & 3.995 & 60 & 4.977 & 78 & 6.237 & 96 & 8.630 \\
4 & 0.911 & 23 & 2.668 & 42 & 3.998 & 61 & 5.013 & 79 & 6.377 & 97 & 8.711 \\
5 & 0.970 & 24 & 2.682 & 43 & 4.031 & 62 & 5.184 & 80 & 6.468 & 98 & 8.838 \\
6 & 1.197 & 25 & 2.809 & 44 & 4.075 & 63 & 5.195 & 81 & 6.589 & 99 & 8.862 \\
7 & 1.260 & 26 & 2.919 & 45 & 4.092 & 64 & 5.237 & 82 & 6.679 & 100 & 8.897 \\
8 & 1.263 & 27 & 2.973 & 46 & 4.203 & 65 & 5.248 & 83 & 6.947 & 101 & 8.944 \\
9 & 1.282 & 28 & 3.100 & 47 & 4.317 & 66 & 5.333 & 84 & 7.064 & 102 & 8.993 \\
10 & 1.337 & 29 & 3.105 & 48 & 4.365 & 67 & 5.431 & 85 & 7.065 & 103 & 9.032 \\
11 & 1.415 & 30 & 3.113 & 49 & 4.373 & 68 & 5.560 & 86 & 7.354 & 104 & 9.280 \\
12 & 1.468 & 31 & 3.279 & 50 & 4.469 & 69 & 5.593 & 87 & 7.387 & 105 & 9.327 \\
13 & 1.549 & 32 & 3.338 & 51 & 4.470 & 70 & 5.642 & 88 & 7.432 & 106 & 10.234 \\
14 & 1.670 & 33 & 3.371 & 52 & 4.492 & 71 & 5.697 & 89 & 7.486 & 107 & 10.297 \\
15 & 1.841 & 34 & 3.461 & 53 & 4.507 & 72 & 5.816 & 90 & 7.782 & 108 & 10.582 \\
16 & 2.057 & 35 & 3.570 & 54 & 4.512 & 73 & 5.841 & 91 & 7.824 & 109 & 10.868 \\
17 & 2.163 & 36 & 3.664 & 55 & 4.735 & 74 & 5.930 & 92 & 8.042 & 110 & 11.805 \\
18 & 2.294 & 37 & 3.857 & 56 & 4.798 & 75 & 5.961 & 93 & 8.415 & 111 & 12.530 \\
19 & 2.309 & 38 & 3.861 & 57 & 4.808 & & & & & & \\
\hline \hline
\end{tabular}

Table 3: Silmitasertib relative energies $\left(\mathrm{kcal} \cdot \mathrm{mol}^{-1}\right)$ computed at B97-D3BJ/cc-pVTZ//B97-D3BJ/cc-pVTZ theory level.

\begin{tabular}{lc|lc|lc|lc}
\hline \hline Conf. & $\Delta \mathrm{E}$ & Conf. & $\Delta \mathrm{E}$ & Conf. & $\Delta \mathrm{E}$ & Conf. & $\Delta \mathrm{E}$ \\
\hline 1 & 0.000 & 8 & 1.749 & 15 & 5.942 & 21 & 6.749 \\
2 & 0.247 & 9 & 1.749 & 16 & 6.588 & 22 & 7.324 \\
3 & 0.431 & 10 & 1.904 & 17 & 6.588 & 23 & 7.324 \\
4 & 1.521 & 11 & 1.904 & 18 & 6.712 & 24 & 7.489 \\
5 & 1.521 & 12 & 5.384 & 19 & 6.712 & 25 & 7.489 \\
6 & 1.657 & 13 & 5.384 & 20 & 6.749 & 26 & 7.520 \\
7 & 1.657 & 14 & 5.942 & & & & \\
\hline \hline
\end{tabular}


Table 4: Valproic acid relative energies $\left(\mathrm{kcal} \cdot \mathrm{mol}^{-1}\right)$ computed at B97-D3BJ/cc-pVTZ//B97-D3BJ/cc-pVTZ theory level.

\begin{tabular}{lc|ll|ll|ll|ll|lc}
\hline \hline Conf. & $\Delta \mathrm{E}$ & Conf. & $\Delta \mathrm{E}$ & Conf. & $\Delta \mathrm{E}$ & Conf. & $\Delta \mathrm{E}$ & Conf. & $\Delta \mathrm{E}$ & Conf. & $\Delta \mathrm{E}$ \\
1 & 0.000 & 30 & 1.534 & 59 & 2.289 & 88 & 3.061 & 117 & 3.537 & 146 & 4.597 \\
2 & 0.206 & 31 & 1.534 & 60 & 2.328 & 89 & 3.082 & 118 & 3.573 & 147 & 4.597 \\
3 & 0.206 & 32 & 1.561 & 61 & 2.328 & 90 & 3.102 & 119 & 3.573 & 148 & 4.710 \\
4 & 0.427 & 33 & 1.561 & 62 & 2.328 & 91 & 3.102 & 120 & 3.582 & 149 & 4.710 \\
5 & 0.587 & 34 & 1.571 & 63 & 2.328 & 92 & 3.138 & 121 & 3.582 & 150 & 4.956 \\
6 & 0.643 & 35 & 1.571 & 64 & 2.388 & 93 & 3.138 & 122 & 3.583 & 151 & 4.956 \\
7 & 0.643 & 36 & 1.604 & 65 & 2.450 & 94 & 3.149 & 123 & 3.608 & 152 & 5.021 \\
8 & 0.680 & 37 & 1.604 & 66 & 2.450 & 95 & 3.149 & 124 & 3.608 & 153 & 5.021 \\
9 & 0.680 & 38 & 1.622 & 67 & 2.498 & 96 & 3.198 & 125 & 3.618 & 154 & 5.095 \\
10 & 0.729 & 39 & 1.622 & 68 & 2.498 & 97 & 3.198 & 126 & 3.762 & 155 & 5.095 \\
11 & 0.789 & 40 & 1.679 & 69 & 2.527 & 98 & 3.202 & 127 & 3.762 & 156 & 5.144 \\
12 & 0.789 & 41 & 1.679 & 70 & 2.527 & 99 & 3.202 & 128 & 3.925 & 157 & 5.144 \\
13 & 0.934 & 42 & 1.681 & 71 & 2.532 & 100 & 3.205 & 129 & 3.925 & 158 & 5.187 \\
14 & 0.934 & 43 & 1.681 & 72 & 2.532 & 101 & 3.205 & 130 & 4.079 & 159 & 5.187 \\
15 & 1.010 & 44 & 1.724 & 73 & 2.574 & 102 & 3.208 & 131 & 4.079 & 160 & 5.225 \\
16 & 1.010 & 45 & 1.724 & 74 & 2.601 & 103 & 3.233 & 132 & 4.131 & 161 & 5.225 \\
17 & 1.113 & 46 & 1.777 & 75 & 2.601 & 104 & 3.238 & 133 & 4.131 & 162 & 5.435 \\
18 & 1.113 & 47 & 1.777 & 76 & 2.603 & 105 & 3.256 & 134 & 4.194 & 163 & 5.435 \\
19 & 1.166 & 48 & 1.835 & 77 & 2.780 & 106 & 3.256 & 135 & 4.194 & 164 & 5.594 \\
20 & 1.166 & 49 & 1.835 & 78 & 2.780 & 107 & 3.328 & 136 & 4.198 & 165 & 5.594 \\
21 & 1.197 & 50 & 1.857 & 79 & 2.806 & 108 & 3.328 & 137 & 4.198 & 166 & 6.072 \\
22 & 1.197 & 51 & 1.857 & 80 & 2.806 & 109 & 3.340 & 138 & 4.382 & 167 & 6.072 \\
23 & 1.197 & 52 & 1.884 & 81 & 2.826 & 110 & 3.340 & 139 & 4.382 & 168 & 6.732 \\
24 & 1.315 & 53 & 1.884 & 82 & 2.826 & 111 & 3.341 & 140 & 4.416 & 169 & 6.934 \\
25 & 1.315 & 54 & 2.012 & 83 & 2.840 & 112 & 3.341 & 141 & 4.416 & 170 & 6.934 \\
26 & 1.344 & 55 & 2.012 & 84 & 2.907 & 113 & 3.353 & 142 & 4.449 & & \\
27 & 1.344 & 56 & 2.138 & 85 & 2.958 & 114 & 3.353 & 143 & 4.449 & & \\
28 & 1.404 & 57 & 2.138 & 86 & 2.958 & 115 & 3.353 & 144 & 4.450 & & \\
29 & 1.404 & 58 & 2.289 & 87 & 3.000 & 116 & 3.398 & 145 & 4.450 & & \\
\hline \hline
\end{tabular}


putational Drug Repurposing Study," ChemRxiv, DOI: 10.26434/chemrxiv.118ChemRxiv, 22020.

[8] P. Willett, J. M. Barnard, and G. M. Downs, "Chemical similarity searching," Journal of Chemical Information and Computer Sciences, vol. 38, no. 6, pp. 983-996, 1998.

[9] N. Nikolova and J. Jaworska, "Approaches to measure chemical similarity? A review," QSAR \& Combinatorial Science, vol. 22, no. 9-10, pp. 1006-1026, 2003.

[10] A. Bender and R. C. Glen, "Molecular similarity: a key technique in molecular informatics," Org. Biomol. Chem., vol. 2, pp. 3204-3218, 2004.

[11] P. Willett, "Similarity methods in chemoinformatics," Annual Review of Information Science and Technology, vol. 43, no. 1, pp. 3-71, 2009.

[12] P. Willett, "The calculation of molecular structural similarity: Principles and practice," Molecular Informatics, vol. 33, no. 6-7, pp. 403-413, 2014.

[13] G. Maggiora, M. Vogt, D. Stumpfe, and J. Bajorath, "Molecular similarity in medicinal chemistry," Journal of Medicinal Chemistry, vol. 57, no. 8, pp. 3186-3204, 2014.

[14] J. Boström, "Reproducing the conformations of proteinbound ligands: A critical evaluation of several popular conformational searching tools," Journal of Computer-Aided Molecular Design, vol. 15, no. 12, pp. 1137-1152, 2001.

[15] D. S. Wishart, C. Knox, A. C. Guo, D. Cheng, S. Shrivastava, D. Tzur, B. Gautam, and M. Hassanali, "Drugbank: a knowledgebase for drugs, drug actions and drug targets," Nucleic Acids Research, vol. 36, no. suppl 1, pp. D901-D906, 2008.

[16] H. M. Berman, J. Westbrook, Z. Feng, G. Gilliland, T. N. Bhat, H. Weissig, I. N. Shindyalov, and P. E. Bourne, "The Protein Data Bank," Nucleic Acids Research, vol. 28, pp. 235242, 012000.

[17] S. Kim, J. Chen, T. Cheng, A. Gindulyte, J. He, S. He, Q. Li, B. A. Shoemaker, P. A. Thiessen, B. Yu, L. Zaslavsky, J. Zhang, and E. E. Bolton, "PubChem 2019 update: improved access to chemical data," Nucleic Acids Research, vol. 47, pp. D1102-D1109, 102018.

[18] N. C. for Biotechnology Information. PubChem Database, "Chloroquine, cid=2719." https://pubchem.ncbi.nlm.nih.gov/compound/Chloroquine, (accessed on Mar. 23, 2020).

[19] A. D. Ferguson, P. R. Sheth, A. D. Basso, S. Paliwal, K. Gray, T. O. Fischmann, and H. V. Le, "Structural basis of cx-4945 binding to human protein kinase ck2," FEBS Letters, vol. 585, no. 1, pp. 104-110, 2011.

[20] R. Krishnan, A. Tulinsky, G. P. Vlasuk, D. Pearson, P. Vallar, P. Bergum, T. K. Brunck, and W. C. Ripka, "Synthesis, structure, and structure-activity relationships of divalent thrombin inhibitors containing an $\alpha$-keto-amide transition-state mimetic," Protein Science, vol. 5, no. 3, pp. 422-433, 1996.
[21] Parrish, R. M., Burns, L. A., Smith, D. G. A., Simmonett, A. C., DePrince, A. Eugene, Hohenstein, E. G., Bozkaya, Uğur, Sokolov, A. Yu., D. Remigio, Roberto, Richard, R. M., Gonthier, J. F., James, A. M., McAlexander, H. R., Kumar, Ashutosh, Saitow, Masaaki, Wang, Xiao, Pritchard, B. P., Verma, Prakash, Schaefer, H. F., Patkowski, Konrad, King, R. A., Valeev, E. F., Evangelista, F. A., Turney, J. M., Crawford, T. Daniel, Sherrill, and C. David, "Psi4 1.1: An open-source electronic structure program emphasizing automation, advanced libraries, and interoperability," J. Chem. Theory Comput., vol. 13, no. 7, pp. 3185-3197, 2017. PMID: 28489372.

[22] W. L. DeLano and Schrödinger, LLC, "The PyMOL molecular graphics system, version 1.7.0.0.” https://pymol.org, 2015. https://github.com/schrodinger/pymol-open-source.

[23] J. D. Hunter, "Matplotlib: A 2D graphics environment," Comput. Sci. Eng., vol. 9, no. 3, pp. 90-95, 2007.

\section{Supplementary Information}

Note that the conformation numbers reported in the following supplementary tables do not correspond to the same numbered conformations reported in the Results and Discussion sections. This is do to a) the fact that the conformations can change when optimized at different theory level, and b) the lowest energy conformation can also change. 
Table S1: Chloroquine isomer 1's relative energies $\left(\mathrm{kcal} \cdot \mathrm{mol}^{-1}\right)$ computed at HF/6-31G(d)//HF/6-31G(d) theory level.

\begin{tabular}{lc|lc|lc}
\hline \hline Conf. & $\Delta \mathrm{E}$ & Conf. & $\Delta \mathrm{E}$ & Conf. & $\Delta \mathrm{E}$ \\
\hline 1 & 0.000 & 40 & 5.293 & 79 & 8.023 \\
2 & 0.585 & 41 & 5.413 & 80 & 8.113 \\
3 & 0.643 & 42 & 5.726 & 81 & 8.115 \\
4 & 0.837 & 43 & 5.730 & 82 & 8.303 \\
5 & 1.261 & 44 & 5.735 & 83 & 8.403 \\
6 & 1.324 & 45 & 5.781 & 84 & 8.459 \\
7 & 1.436 & 46 & 5.810 & 85 & 8.501 \\
8 & 1.790 & 47 & 5.923 & 86 & 8.691 \\
9 & 1.961 & 48 & 5.936 & 87 & 8.725 \\
10 & 2.256 & 49 & 5.947 & 88 & 8.794 \\
11 & 2.613 & 50 & 6.009 & 89 & 8.845 \\
12 & 2.707 & 51 & 6.016 & 90 & 8.900 \\
13 & 2.764 & 52 & 6.111 & 91 & 8.921 \\
14 & 3.238 & 53 & 6.155 & 92 & 8.923 \\
15 & 3.416 & 54 & 6.201 & 93 & 9.028 \\
16 & 3.481 & 55 & 6.268 & 94 & 9.065 \\
17 & 3.518 & 56 & 6.483 & 95 & 9.097 \\
18 & 3.906 & 57 & 6.568 & 96 & 9.198 \\
19 & 4.006 & 58 & 6.648 & 97 & 9.221 \\
20 & 4.114 & 59 & 6.650 & 98 & 9.364 \\
21 & 4.167 & 60 & 6.678 & 99 & 9.703 \\
22 & 4.393 & 61 & 6.692 & 100 & 10.016 \\
23 & 4.429 & 62 & 6.754 & 101 & 10.020 \\
24 & 4.466 & 63 & 6.881 & 102 & 10.307 \\
25 & 4.589 & 64 & 6.922 & 103 & 10.633 \\
26 & 4.622 & 65 & 7.075 & 104 & 10.634 \\
27 & 4.643 & 66 & 7.093 & 105 & 10.765 \\
28 & 4.652 & 67 & 7.164 & 106 & 10.817 \\
29 & 4.656 & 68 & 7.205 & 107 & 11.006 \\
30 & 4.858 & 69 & 7.208 & 108 & 11.069 \\
31 & 4.870 & 70 & 7.308 & 109 & 11.359 \\
32 & 4.909 & 71 & 7.358 & 110 & 11.497 \\
33 & 4.935 & 72 & 7.472 & 111 & 11.529 \\
34 & 5.077 & 73 & 7.494 & 112 & 11.870 \\
35 & 5.146 & 74 & 7.498 & 113 & 11.901 \\
36 & 5.156 & 75 & 7.505 & 114 & 13.385 \\
37 & 5.244 & 76 & 7.793 & 115 & 13.643 \\
38 & 5.262 & 77 & 7.942 & 116 & 13.989 \\
39 & 5.277 & 78 & 8.002 & & \\
\hline \hline & & & & & \\
\hline
\end{tabular}

